\title{
Impact of cross-enterprise data sharing on portable media with decentralised upload of DICOM data into PACS
}

\author{
K. Y. E. Aryanto • R. van de Wetering • A. Broekema • \\ Peter M. A. van Ooijen • M. Oudkerk
}

Received: 11 September 2013 /Revised: 11 October 2013 / Accepted: 17 October 2013 /Published online: 17 November 2013

(C) The Author(s) 2013. This article is published with open access at Springerlink.com

\begin{abstract}
Objectives To evaluate portable media utilisation for image data sharing between enterprises. To predict the costs required to keep up with the trend. To identify related problems.

Methods A software package was developed to include patient image data from CD into our normal workflow. The trend in the workload of CDs that were uploaded into a Picture Archiving and Communication System (PACS) over 89 months was analysed. The average number of images per month (and per investigation) was calculated to provide the estimation of storage and cost required in the whole process. Results All Digital Imaging and Communications in Medicine (DICOM) files can be read from compact disc (CD) on any workstation in the hospital, processed quickly to the central server and checked after storage using the software tool. A total of 33,982,404 images from 88,952 CDs have been stored into the PACS system. In recent years, the stored images have reached an average of 4.2 terabytes (TB) uncompressed annually.

Conclusion Integrated information about patients is clearly needed to provide easy and timely access to these data. The steadily growing storage can be solved by a more automated approach to portable media handling or the installation and
\end{abstract}

K. Y. E. Aryanto $\cdot$ P. M. A. van Ooijen $(\bowtie) \cdot$ M. Oudkerk

Department of Radiology, Center for Medical Imaging - North East

Netherlands (CMINEN), University of Groningen, University

Medical Center Groningen, PO BOX 30001, 9700 RB Groningen,

The Netherlands

e-mail: p.m.a.van.ooijen@umcg.nl

R. van de Wetering

Strategy and Operations, Deloitte Consulting BV, Utrecht,

The Netherlands

A. Broekema

Department of Radiology, University Medical Center Groningen, Groningen, The Netherlands acceptance of network-based transfer using cross-enterprise document sharing (XDS).

Key points

- Rapid assimilation of external imaging into a PACS system is essential.

- But data distribution using portable media also carries some disadvantages.

- A DICOM data uploader incorporates studies from portable media to hospital workflow.

- Automated media handling or XDS should solve the steadily growing storage problem.

- Software improvements will facilitate the steady increase in the amount of CDs processed.

Keywords Data sharing - Information distribution ·

CDROM · PACS (Radiology) · Radiology information system

$\begin{array}{ll}\text { Abbreviations } \\ \text { DICOM } & \text { Digital Imaging and Communications in Medicine } \\ \text { PACS } & \text { Picture Archiving and Communication System } \\ \text { CD } & \text { Compact disc } \\ \text { DVD } & \text { Digital versatile disc } \\ \text { XDS } & \text { Cross-enterprise document sharing } \\ \text { XDS-i } & \text { Cross-enterprise document sharing for imaging } \\ \text { IHE } & \text { Integrating the Healthcare Enterprise } \\ \text { PDI } & \text { Portable data for imaging } \\ \text { ftp } & \text { File transfer protocol } \\ \text { GB } & \text { Gigabyte } \\ \text { TB } & \text { Terabyte } \\ \text { FTE } & \text { Full-time equivalent }\end{array}$

\section{Introduction}

The need for sharing data and the introduction of digital imaging in radiology have prompted the use of portable media 
(e.g. recordable compact disc $[\mathrm{CD}]$ and digital versatile disc [DVD]) to distribute patient data. Exchange of Digital Imaging and Communications in Medicine (DICOM) data using such media has been defined in the DICOM standard, including the requirements of the storing formats, structure and profiles [1-3]. Meanwhile, the Integrating the Healthcare Enterprise (IHE) initiative [4] provides a profile of portable data for imaging (PDI) for the necessary standardisation for portable data exchange [5].

Distribution of patient data using recordable CDs is beneficial in terms of cost and shipping when compared with the previous situation using physical hard copy film [6, 7]. It prevents loss of studies compared with the film-based distribution and provides the patients with the possibility of a selfmaintained archive of their own imaging records which is claimed to improve patient care [7].

Although portable media offer benefits, they also have some disadvantages. The actual viewing of images becomes one of the main problems, since each vendor provides its own viewer on the $\mathrm{CD}$. This hampers the evaluation of the image data by inexperienced users. The DICOM reader and information on the origin of the data that are included in the media make the data vulnerable to alteration, which could be considered as an unsafe method to distribute DICOM images [8]. Furthermore, local installation of a software package to view the data from portable media is prohibited in most hospitals due to security reasons and patient privacy aspects. Different policies and workarounds of handling data in each hospital, such as a hospital-specific identification (ID), also disrupts the interchange of imaging data [9].

The uncertainty of IHE PDI compliance can also challenge the reliability of image distribution. Compliance of digital media with the IHE PDI is only achieved by less than a quarter of the heath institutions in the United States (U.S.) [10]. Kalia et al. [10] mentioned that the uncertainty of IHE PDI compliance will hamper image availability in the Picture Archiving and Communication System (PACS) of receiving institutions with a strict policy of importing images provided by outside institutions.

To tackle the problems stated above, a system was designed and implemented that incorporates the study data from the CDs into the normal hospital workflow using a decentralised upload of the CD data [11]. The outcome is a new procedure for external image data at our institution, which was first introduced in August 2005. This procedure incorporates the DICOM image data on CDs from other institutions into our normal workflow either on the institutional web server or on the radiology PACS. This procedure enables the clinical physicians to use the hospital's viewers embedded into their electronic medical record (EMR) to access the imported images and not necessarily require separate query to the PACS or a new image viewer they are not used to. Literature and practice show that importing the image data from CDs into the local PACS leads to a significant reduction of imaging examination repetition, for example in the emergency department [12]. A novelty in our set-up was that the CDs remain at the requesting department and are uploaded to a central service at the radiology department, where they are handled further for inclusion into the normal workflow. By implementing the upload in a decentralised way, the CDs remain available for review at the requiring department; $\mathrm{CDs}$ are not brought to radiology or sent there by internal mail and stacks of CDs at the radiology department are avoided. At the time of implementation, assumptions were made about the number of CDs that will have to be processed. However, with several subsequent improvements in the software tools used for this task, both the interest in this possibility and the amount of CDs processed steadily increased.

In this study we describe the current procedures and software tools used and then evaluate the status of the number of CDs and images uploaded using this procedure for over 7 years. Furthermore, we discuss the pitfalls and consequences of the upload of data on CD.

\section{Materials and methods}

An in-house software package called DICOMUploader (DIUP) was developed in order to include patient image data from CDs into our normal workflow. This software package was the result of improvement of an earlier version of the uploading procedure [13].

DIUP reads all DICOM files from a CD on any workstation in the hospital. After loading the $\mathrm{CD}$, the patient name and the available series are displayed. The physician can choose the relevant series and push these to a file transfer protocol (ftp) server after providing the in-house patient ID and checking whether the selected data are correct. This forces the physician to check whether the patient is registered at our hospital, and if not, to register the patient first. Replacement of the patient ID with the in-house patient ID is performed during the transfer to the central ftp server. At the radiology department, the data are retrieved from the ftp archive and stored at the appropriate DICOM node. During the storage process, the information regarding a patient having external data is recorded. The uploaded images will be updated with patient identifiers from our institution's radiology information system (RIS) by overwriting the original patient ID and accession number with the correct patient ID and accession number in the DICOM header. Image-related materials such as radiology reports or informed consents are obtained from other institutions via email or fax. These image-related materials were not stored in the system but delivered separately to the physicians. Later, the stored data are subsequently removed from the ftp site if the transfer is successfully finished. Using DIUP, physicians can also track whether their $\mathrm{CD}$ has already been stored into the 
DICOM node, thus avoiding resending the same CD over and over.

Besides data handling, DIUP also registers all actions and stores them in a database. This database can be used by the requester of the upload to check on progress or, in the case of this study, to analyse the usage of the software.

In this study we analysed the trend in the workload of CDs that were uploaded per month from August 2005 until December 2012 ( 89 months) by querying data recorded in the database of DIUP. Based on the results of these queries, the amount of storage required was calculated using an average size per image. The average image size was determined for each type of investigation (computed tomography [CT], magnetic resonance $[\mathrm{MR}]$, combined positron emission tomography $[\mathrm{PET}] / \mathrm{CT}$, etc.). Furthermore, there was an estimate on the cost involved in this whole process.

Moreover, a short written survey was sent to heads of departments, heads of laboratories, radiologists, PACS application administrators or information and communication technology (ICT) staff from 39 Dutch hospitals to determine the common practice for cross enterprise data sharing. We obtained contact details from secretaries of each individual radiology department and through our own network. We sent out a total of 39 requests to all 39 hospitals; therefore each hospital had one of their representatives to fill in the questionnaire.

The respondents were asked to fill out the survey online and a reminder was sent to all the respondents who had not yet returned a questionnaire. Eight questions were asked regarding the practice and future movement of respondent institutions towards image sharing and their current use of portable media to distribute medical images. The questions are listed below.

1. Does your institution share image data with other healthcare institutions?
a. Yes (Continue to question number 2)
b. No (Continue to question number 6)

2. Which method does your institution use for sharing such data?
a. CD/DVD (Continue to question number 3)
b. Other portable media (Continue to question number 6)
c. XDS (Continue to question number 7)
d. Other :

3. How many CD/DVD does your institution produce and send per week?

4. How many CD/DVD arrive at your institution per week?

5. Are incoming CDs/DVDs uploaded or saved into your own digital archive/PACS system?
a. Yes
b. No
c. Don't know

6. Are you planning on moving to a network-based solution such as XDS?
a. Yes
b. No
c. Don't know

7. If No, why not?

8. Remarks

The responds to the questionnaires were decoupled from the e-mail address of the participant and thus anonymised at the time of submission of the response.

\section{Results}

\section{DIUP results}

A total of 33,982,404 images from 88,952 CDs have been stored into the PACS of our hospital over the 89-month period. The number of images transferred per year steadily increased since the first year of the use of DIUP (Fig. 1). In 2012, the system has transferred nearly 9 million images from multiple different modalities; most data stored originated from CT and MR; ultrasound (US) and computed radiography (CR) examinations were also commonly uploaded (Fig. 2).

The number of external CDs handled with this procedure increased over the 89 months period in steps from less than 600 CDs uploaded each month in the 1st year to an average of more than 1,300 CDs uploaded per month in the last year (Figs. 3 and 4). The yearly upload of CDs shows an annual increase of 1,500-2,000 CDs, with a current total of 18,000 CDs in 2012 (Fig. 5).

Roughly, an average of 60 gigabytes (GB) of uncompressed images per month or $720 \mathrm{~GB}$ of uncompressed images per year were added into the PACS in the early stages of using DIUP. In 2012, the amount of images stored through the DIUP reached $360 \mathrm{~GB}$ uncompressed per month or an average of 4.2 terabyte (TB) uncompressed per year, which accounts for approximately $28 \%$ of the total current storage per year on our PACS environment.

\section{Other institutions}

We conducted a survey in 39 Dutch hospitals to determine the extent of the use of portable media and whether there is a plan for moving into the latest technology. In total, 14 questionnaires were filled in online, each came from a different institution. This is a response rate of $36 \%$. All the completed questionnaires were included into the analysis, subject to quality criteria (i.e. no missing indicators and values).

Twelve out of 14 respondents stated that their hospital was still using CD/DVD as the method of data sharing, while three of them also used other alternative portable media to share 
Fig. 1 Total images transferred using DIUP annually
Images transferred per year using DIUP

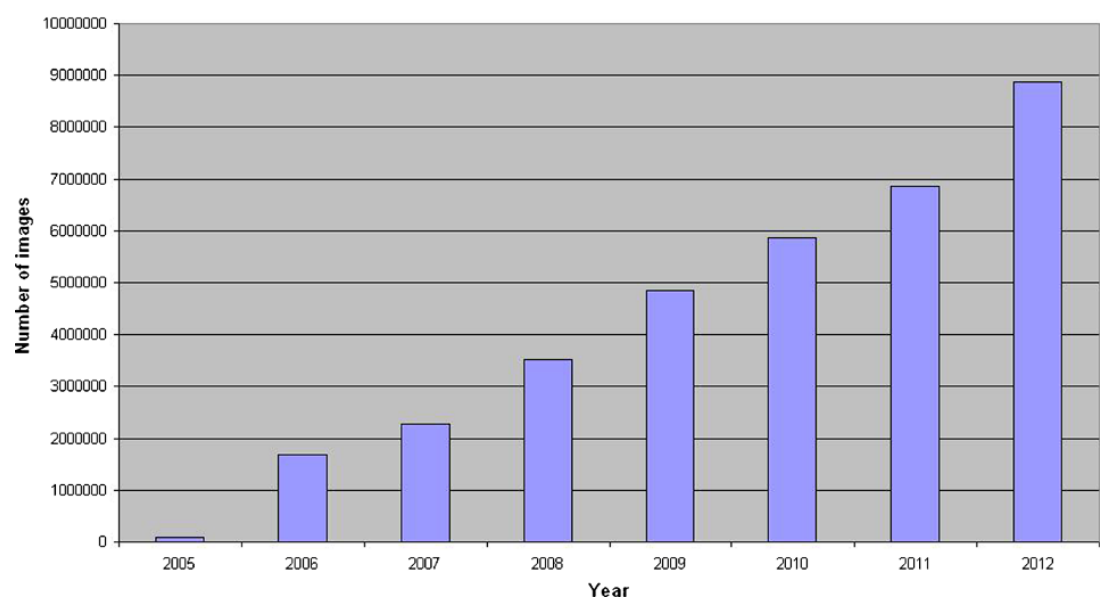

data (Table 1). The use of network-based methods is still rare, shown by only one hospital that already implemented crossenterprise document sharing (XDS), but also still utilise the CD/DVD method for image sharing. This mainly caused by the fact that not all other cooperating institutions are able to connect to the XDS environment.

The survey indicates that at least $60 \mathrm{CDs} / \mathrm{DVDs}$ arrive and $75 \mathrm{CDs} / \mathrm{DVD}$ s are produced every week in a hospital and reaches up to an arrival of $425 \mathrm{CDs}$ and to a production of $200 \mathrm{CDs}$ per week in a large university hospital (Fig. 6).

All hospitals currently using CD/DVD for data transfer uploaded the incoming images to their PACS. Almost all institutions stated that they are planning on moving into a network-based solution, such as XDS/XDS-I in the near future (Table 2).

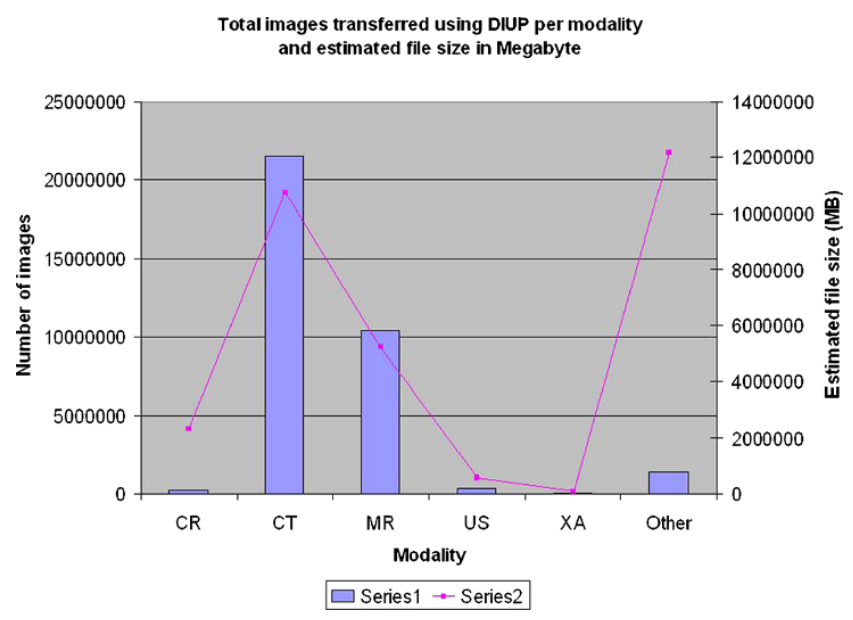

Fig. 2 Total images transferred using DIUP per modality and estimated file size in megabytes ( $C R$ computed radiography, $C T$ computed tomography, $M R$ magnetic resonance, $U S$ ultrasound, $X A$ X-ray angiography and Other modalities including nuclear medicine, digital radiography, radio-fluoroscopy, etc.)

\section{Discussion}

The results presented in this study clearly show an enormous increase in the amount of data shared between healthcare institutions. The increased use of the upload of data from portable media into the hospital infrastructure shows that it is providing an important service to the hospital. However, although data sharing is beneficial in improvement of quality of patient care and should be done when needed, the amount of data shows that without a software tool as described above, the handling of the CDs with patient data will become virtually impossible.

Even though uploading images from CDs into PACS is likely to reduce the rate of repeat imaging in one institution [14], a centralised upload into PACS by the radiology department, as mainly implemented by most PACS vendors, is often leading to large stacks of CDs and very high workload. By providing a decentralised upload, the workload is divided over multiple departments. The time required to perform the $\mathrm{CD}$ handling will be approximately the same regardless of who does the upload. However, the time required with centralised upload to take care of internal shipping of the media and keeping track of the progress of the handling is saved when using decentralised upload.

An additional benefit of the decentralised upload is that the CD remains at the point of care, which means that in case of an emergency situation requiring access to the image data before upload process has finished, the image data remains available at the point of care by using the original CD/DVD.

Previous evaluation showed that the procedure developed at our institution using decentralised uploading of the data works very efficiently and allows easy access to the data [13]. This is indicated by the satisfaction from the respondents in the referred work where $63 \%$ rated the procedure as excellent, compared with the manual procedure, while the rest marked the system as good. The growing use has shown a wide scale adoption of the method throughout the hospitals, thus 
Fig. 3 Number of CDs transferred in the first 12 months (August 2005 to July 2006)
Number of CDs transferred in the first year

(August 2005 - July 2006)

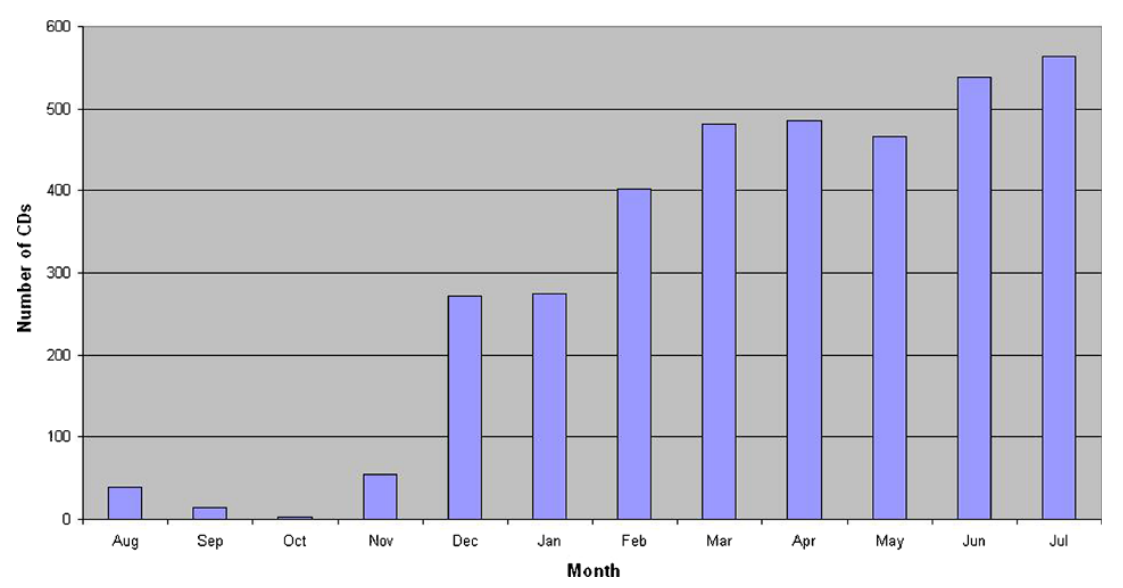

suggesting a preferred procedure compared with reading directly from $\mathrm{CD} / \mathrm{DVD}$.

However, although the utility of our system with decentralised uploading is already high and reduces part of the workload, further enhancements are still possible. The automatic entries of the studies to the radiology information system and an even more automatic handling of the CDs are currently under development to decrease the time required for handling the CDs at our institution. This automation will save time and money since less human resources are involved and most of the procedures are fully taken care of by the system.

During our 7 years of experience using portable media for sharing data, several issues have emerged. Examples are:

1. Read failure of CDs.

This was mainly caused by physical defects on the media, such as scratches, corrosion or failure in writing data at the host institutions.

2. Problem reading data on $\mathrm{CD}$.

Differences in how the information systems at various institutions handled the data might have caused these mismatches in reading patient data. In some cases, DIUP cancelled processing of the related patient or study data causing the image data to be skipped and requiring a subsequent manual upload of the data.

3. Update of external components.

Changes in hospital systems and updates or changes of standards (e.g. DICOM) could cause failures in handling the data and therefore require an update of the DIUP application.

4. Problems with resend of old data.

Resending the complete history of a patient on a new $\mathrm{CD}$ at a subsequent visit often occurs. In such cases, the old data will be ignored and not stored into PACS. However, problems with access, importability and viewing may then cause a delay in patient care [10].

The survey that was conducted in several hospitals may be biased because of the lack of intra-institutional consensus within respondents' institutions in filling-in the questionnaire. However, such a problem should not disrupt the reliability of the given responses since the questions were designed to
Fig. 4 Number of CDs transferred in 2012

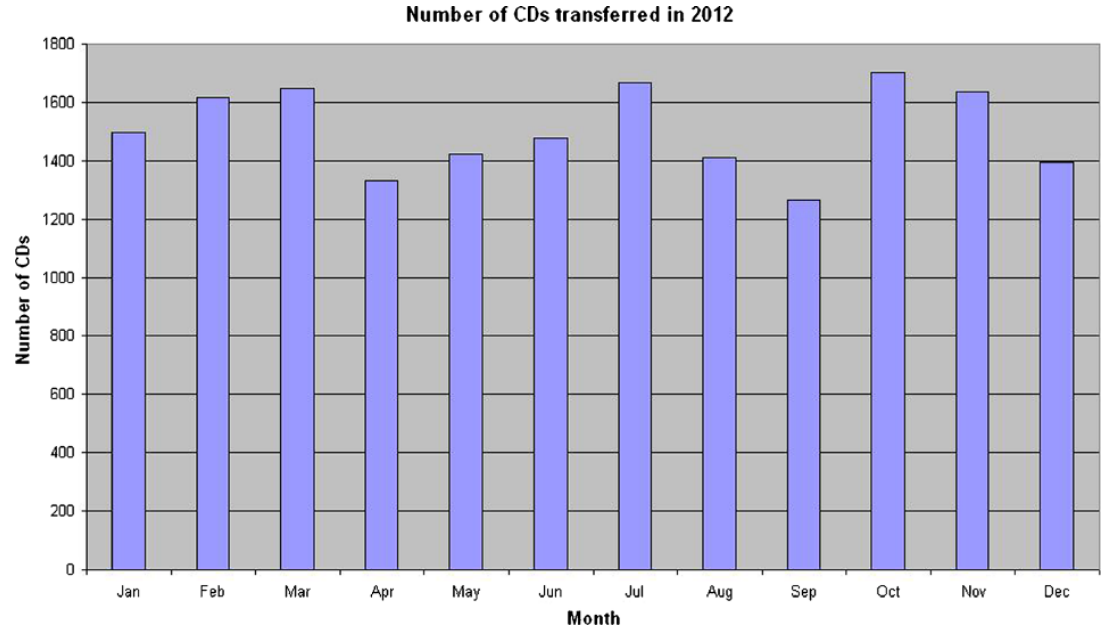


Fig. 5 Total number of CDs transferred using DIUP
Total number of CDs transferred using DIUP until December 2012

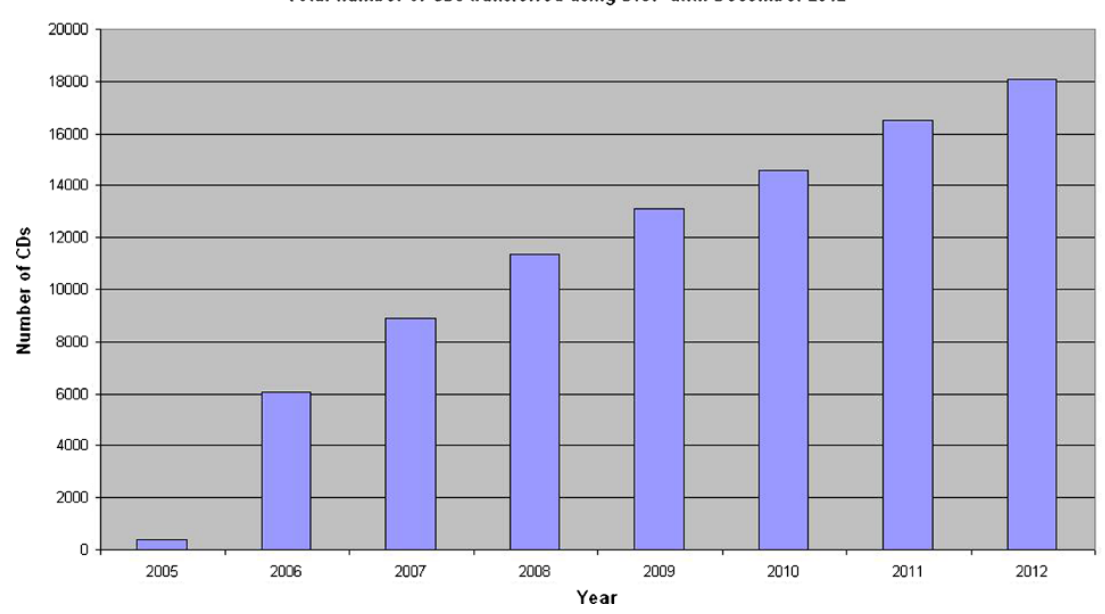

obtain certain answer from one institution despite the profession of the appointed person.

Besides the problems due to the number of CDs shipped between hospitals, the storage of all these data is the next problem to be tackled. The ever-increasing amount of incoming data is unavoidable. The $4.2 \mathrm{~TB}$ per year of images with the incremental amount of 1,500-2,000 CDs uploaded per year not only puts a heavy burden on the local PACS storage capacity (in our case $28 \%$ of the total storage per year) but may also cause other problems in the future, such as time consumed in searching and processing data or disruption in data registration in the information system, if this issue is not handled properly.

A general problem inherent to the existence of a local copy of the data at different hospitals is that it will hamper a proper audit trail of the data. This could increase the risk of patient privacy violation and data security breaches, and thus decrease data integrity. Shipping the CDs between hospitals will lead to multiple copies of the imaging data at multiple sites without any guarantee that the copies are and will remain identical. Since portable media such as CD or DVD are still used for data exchange by a majority of the hospitals ( $85 \%$ of the respondent hospitals) and the incoming CD/DVDs are all uploaded into their images archive system, this will lead to fast growing storage problems.

Excluding the labour costs for handling the CDs and uploading the data, the cost of media and shipping per $\mathrm{CD}$ will be about $€ 1.90$ per CD/DVD. This breaks down to $€ 0.50$ for the medium, $€ 0.30$ for the jewel case, $€ 0.10$ for the

Table 1 Methods used by institutions for sharing data

\begin{tabular}{lrrl}
\hline Method & Total & Percentage & Total respondents \\
\hline CD/DVD & 12 & $85.71 \%$ & 14 \\
Other & 4 & $28.57 \%$ & \\
XDS & 1 & $7.14 \%$ & \\
\hline
\end{tabular}

envelope and $€ 1.00$ for shipping. Based on this estimation, the total cost per month can currently be set at $1,600 \times € 1.90=$ $€ 3,040$. This yields an annual cost for media and shipping only of $€ 36,480$. This cost is only for the CDs getting into our institution and thus covered by the sending party; our institution has to pay for the internal handling of the CDs. In comparison, the number of CDs currently produced by our institution is about $600 \mathrm{CDs}$ per month, resulting in an annual cost of $€ 13,680$.

Labour cost for the handling of the CDs coming into our hospital using our current procedure is about $10 \mathrm{~min}$; this consists of $3 \mathrm{~min}$ for the requesting party at the point of care and $7 \mathrm{~min}$ at the radiology department. However, the production and shipping of a CD is also estimated to be approximately $10 \mathrm{~min}$ of labour, including preparation for shipping ( $\mathrm{CD}$ in jewel case, jewel case into envelope, address filling, and then sending). This means in our institution with 1,600 incoming CDs per month and 600 outgoing CDs per month, this will add up to $4,400 \mathrm{~h}$ per year, equalling about 2.3 fulltime equivalents (FTEs).

Developments such as the XDS/XDS-i profiles of the Integrating the Healthcare Enterprise (IHE) initiative [15]

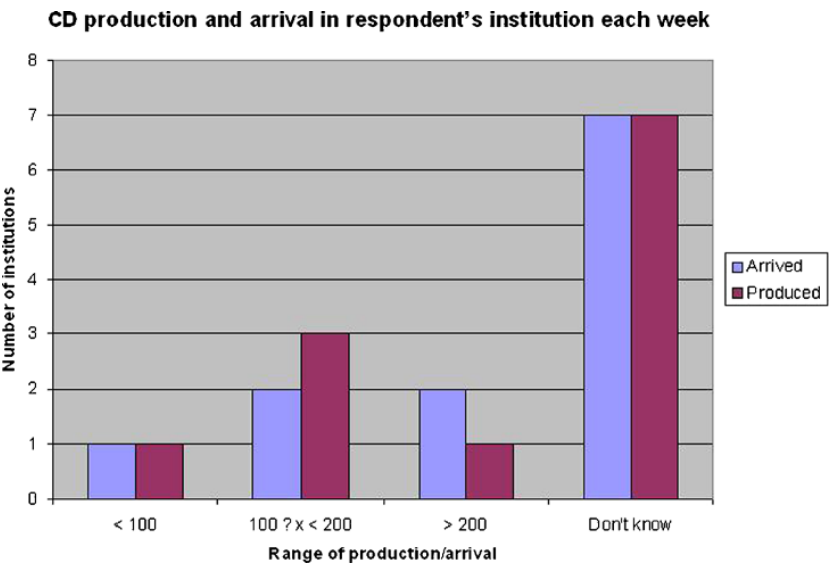

Fig. $6 \mathrm{CD}$ production and arrival in respondent's institution each week 
Table 2 Planning on moving to network based solution

\begin{tabular}{lrr}
\hline XDS migration & Total & Percentage \\
\hline Yes & 12 & $85.71 \%$ \\
No & 1 & $7.14 \%$ \\
Don't know & 1 & $7.14 \%$ \\
\hline
\end{tabular}

could solve part of the problem by real-time sharing data between healthcare institutions over the network $(85 \%$ of the respondents are planning on moving to a network-based solution such as XDS for their future data sharing). XDS is currently often implemented as a replacement of the CD transfer by still retaining local copies of the image data. It would be better to implement XDS the way in which it was designed by really sharing image data on demand with the original data remaining at the source and no local copies. However, in most cases, the inter-institutional infrastructure is insufficient to allow fast, accurate and reliable shared access. Implementing XDS should strongly reduce the amount of hours required for handling, reading and burning CDs.

In our survey, only one respondent mentioned that they implemented the XDS environment for their image sharing with no further information about their counterparts. There are two possibilities why the counterparts were not included: either the counterpart did not reply to the questionnaire or it was an institution outside the scope of our survey. From the reply of this one respondent using XDS, it can be deduced that more hospitals have implemented XDS. Furthermore, it will be an incentive to engage collaborating institutions to connect and also implement XDS. For example, our own institution is also moving towards the implementation of XDS.

Looking at the number of FTEs shown in the results above will lead to a less labour-intensive handling of XDS instead of the $\mathrm{CD}$ upload, since the disc operator is no longer needed. Furthermore, the media costs involved will be strongly reduced and local storage of the data is no longer required. These factors should be sufficient to make the business case for XDS.

Data sharing using methodologies such as XDS can be considered part of a higher level of PACS maturity in compliance with the PACS maturity model (PMM) [16] that describes PACS maturity and potential evolution in the hospital enterprise. Thus, it can be a cost-saving solution by reducing the expenses used during the transfer of data and a critical next step in the evolution of PACS in the hospital enterprise.

Yet, this solution will raise several issues to consider. For example, should the storage be located in separate sites of origin or would it be better to have a large central back-up storage facility at one site only? How long will or should data be kept in other sites than the owners' site? And again only data within the affinity domain can be seen, where data outside that domain still will rely on portable media of any kind. The answer to those questions will determine what the best implementation is and later what will be the costs of the system itself.

In conclusion, there is a clear need for integration of information from patients acquired at other hospitals into the normal workflow to provide easy and timely access to patient data. However, distributing images using portable media may consume more than a quarter of the total capacity of the institutional image storage to be filled up by external data. Therefore, the need for storage of external data into the local PACS is steadily growing and will use even more resources at considerable costs. Solutions for this are to be found in a more automated approach to the $\mathrm{CD}$ handling in combination with installation and acceptance of network-based sharing using XDS between hospital enterprises.

Acknowledgment The authors would like to thank Dr. E.J.K. Noach for her editorial help in preparing the manuscript.

Funding This work is part of the ENACT project which is funded by the ZonMw Innovative Medical Devices Initiative (IMDI) call under project registration number 104002003 .

Open Access This article is distributed under the terms of the Creative Commons Attribution License which permits any use, distribution, and reproduction in any medium, provided the original author(s) and the source are credited.

\section{References}

1. DICOM Standard Committee (2011) Part 10: Media Storage and File Format for Media Interchange (PS 3.10-2011) in DICOM Standard. Available via http://medical.nema.org/Dicom/2011/11_10pu.pdf. Accessed 6 August 2013.

2. DICOM Standard Committee (2011) Part 11: Media Storage Application Profiles (PS 3.11-2011) in DICOM Standard. Available via http://medical.nema.org/Dicom/2011/11_11pu.pdf. Accessed 6 August 2013.

3. DICOM Standard Committee (2011) Part 12: Media Formats and Physical Media for Media Interchange (PS 3.12-2011) in DICOM Standard. Available via http://medical.nema.org/Dicom/2011/11 12pu.pdf. Accessed 6 August 2013.

4. IHE International (2013) Integrating the Healthcare Enterprise. Available via http://www.ihe.net/. Accessed 6 August 2013.

5. IHE International (2012) Portable Data for Imaging Integration Profile in IHE Radiology Technical Framework Volume 1 Section 15. Available via http://www.ihe.net/Technical_Framework/ upload/IHE_RAD_TF_Vol1.pdf. Accessed 6 August 2013.

6. Flanders $\mathrm{AE}(200 \overline{9})$ Medical image and data sharing: are we there yet? Radiographics 29(5):1247-1251. doi:10.1148/rg.295095151

7. Mehta A, Dreyer K, Thrall J (1999) Enhancing availability of the electronic image record for patients and caregivers during follow-up care. J Digit Imaging 12(Suppl 1):78-80. doi:10.1007/BF03168762

8. McEvoy FJ, Svalastoga E (2009) Security of patient and study data associated with DICOM images when transferred using compact disc media. J Digit Imaging 22(1):65-70. doi:10.1007/ s10278-007-9068-x

9. Kuiper JW, Broekman K, De Baat L (2004) A new outpatient with PACS data on CD, now what? In: Inchingolo P, Pozzi- 
Mucelli R (eds) EuroPACSMIR 2004 in the enlarged Europe. EUT, Trieste

10. Kalia V, Carrino JA, Macura KJ (2011) Policies and procedures for reviewing medical images from portable media: survey of radiology departments. J Am Coll Radiol 8(1):39-48. doi:10.1016/j.jacr.2010.07. 007

11. Van Ooijen PMA, Guignot J, Mevel G, Oudkerk M (2005) Incorporating out-patient data from CD-R into the local PACS using DICOM worklist features. J Digit Imaging. doi:10.1007/s10278005-5158-9

12. Sodickson A, Opraseuth J, Ledbetter S (2011) Outside imaging in emergency department transfer patients: CD import reduces rates of subsequent imaging utilization. Radiology 260(2):408-413. doi:10. 1148/radiol.11101956
13. Van Ooijen PMA, Roosjen R, De Blecourt MJ, Van Dam R, Broekema A, Oudkerk M (2006) Evaluation of the use of CDROM upload into the PACS or institutional web server. J Digit Imaging. doi:10.1007/s10278-006-0932-x

14. Lu MT, Tellis WM, Fidelman N, Qayyum A, Avrin DE (2012) Reducing the rate of repeat imaging: import of outside images to PACS. AJR Am J Roentgenol 198(3):628-634. doi:10.2214/AJR.11.6890

15. IHE Wiki (2013) Cross-enterprise Document Sharing for Imaging. Available via http://wiki.ihe.net/index.php?title=Cross-enterprise Document_Sharing for_Imaging. Accessed 6 August 2013

16. Van De Wetering R, Batenburg R (2009) A PACS maturity model: a systematic meta-analytic review on maturation and evolvability of PACS in the hospital enterprise. Int J Med Inform 78(2):127-140. doi:10.1016/j.ijmedinf.2008.06.010 